\title{
Study of shear force as a distance regulation mechanism for scanning near-field optical microscopy
}

\author{
C. Durkan ${ }^{\text {a) }}$ and I. V. Shvets \\ Department of Physics, Trinity College Dublin, Ireland
}

(Received 31 May 1995; accepted for publication 27 September 1995)

\begin{abstract}
Results of the investigation into the suitability of the shear-force distance regulation mechanism for scanning near-field optical microscopy (SNOM) are presented. It is shown that there is a range of relative sizes of surface features to tip size that gives rise to malfunctions of the shear-force distance regulation mechanism. If the size of the tip is comparable to the size of any depressions on the sample, the corresponding shear-force image may show contrast reversal. It is also shown that the resolution obtained when imaging a surface with the shear-force scanning microscope may differ for the two perpendicular lateral directions. The resolution along the oscillation axis is usually lower than the one perpendicular to it. This has implications for SNOM images. Therefore, the interpretation of shear force, and hence SNOM images, may become complex when imaging any sample with significant surface roughness. (C) 1996 American Institute of Physics. [S0021-8979(96)10001-8]
\end{abstract}

\section{INTRODUCTION}

Shear-force distance regulation has practically become a standard component of most scanning near-field optical microscopes (SNOMs) today. ${ }^{1,2}$ Its development was primarily driven by the need to have a distance regulation mechanism for SNOM, to enable the imaging of nonconducting, as well as conducting surfaces. This distance control mechanism is particularly suited to SNOM probes, which typically consist of tapered optical fiber tips. ${ }^{3}$ These fiber tips have relatively small lateral spring constants $(0.1-300 \mathrm{~N} / \mathrm{m})$, and larger longitudinal ones, in contrast to cantilevered atomic force microscope (AFM) tips, which are the opposite. ${ }^{4,5}$ As a result, they are more sensitive to shearing forces. The fiber tip is oscillated laterally by a few nm near mechanical resonance. If the tip approaches a sample this then gives rise to shearing forces of a few $\mathrm{nN}$, which can sufficiently shift the resonance and damp the oscillations. The nature of the shear force is not well established. It could be an effect combining air damping, capillary forces, van der Waals forces, and image current dissipation. The proximity range over which the oscillations are damped to zero scales with the oscillation amplitude. For instance, for a $10 \mathrm{~nm}$ oscillation amplitude, the distance over which this is damped to zero is approximately $10 \mathrm{~nm}$. The effect of shear force is readily measurable. The distance at which shear force comes into effect is of the right order for SNOM. ${ }^{6}$ Therefore, shear force is commonly used for distance regulation in SNOM. In the present work, we decided to study the operation of shear-force distance regulation and especially analyze its applicability for SNOM imaging on surfaces with roughness.

\section{EXPERIMENT}

The fiber tips are produced using a home made computer-controlled pulling machine. ${ }^{7}$ The principle of this is somewhat similar to commercial micropipette pullers ${ }^{8}$ (see

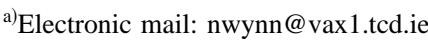

Fig. 1). It consists of a $20 \mathrm{~W} \mathrm{CO}_{2}$ laser for heating the fiber, and a $90 \mathrm{~W}$ dc servomotor for subsequent stretching. The pulling mechanism incorporates a feedback system based on an optical encoder for reproducible pulls. The pulling parameter's velocity and acceleration are thus very well controlled. The laser is pulsed using the computer, and there is the possibility to make as many pulses as required, for any duration down to $5 \mu \mathrm{s}$. We typically use one pulse, of duration $10 \mathrm{~ms}$, during which the pull is initiated. There are two tapered regions, typically of overall length $200 \mu \mathrm{m}$. The acceleration we use is of the order of $10 \mathrm{~m} \mathrm{~s}^{-2}$. The shape of the fiber tips is critically dependent on the pulling parameters.

At least $60 \%$ of the tips made are suitable for SNOM. The tips are then vacuum coated with $100-150 \mathrm{~nm}$ of aluminum, leaving an aperture at the end, of diameter in the range $40-150 \mathrm{~nm}$ (see Fig. 2).

These tips are then mounted with a free end of approximately $2-3 \mathrm{~mm}$, giving rise to resonance frequencies in the range $10-15 \mathrm{kHz}$ with $Q \sim 30$ and $k \sim 100-300 \mathrm{~N} / \mathrm{m}$ for the main body of the fiber. The spring constant for the tip region itself, which is $200 \mu \mathrm{m}$ long, can be much smaller.

The shear-force detection is based on shadowing of a laser beam by the edge of the oscillating fiber. The detection beam is delivered to the tip by means of a single-mode optical fiber. This produces a relatively well defined spot at the tip edge about $10 \mu \mathrm{m}$ in diameter. This spot is placed about $300 \mu \mathrm{m}$ from the end of the tip, just before the tapered region. The principle is illustrated in Fig. 3. The shadowed beam is detected with a photodiode, and lock-in detection is used thereafter. An oscillation amplitude of the SNOM tip by $10 \mathrm{~nm}$ peak-peak is thus easily detectable, with a signalnoise ratio of about 10. As mentioned in Sec. I, the range over which this oscillation becomes damped to zero scales with the oscillation amplitude. Some approach curves are shown in Fig. 4. The distance dependence of the oscillation amplitude is approximately exponential, and can be fitted with $R[1-\exp (-a z)]$. The feedback signal is fed directly into the log amplifier of our scanning tunneling microscopy (STM) unit. The signal used for feedback is 

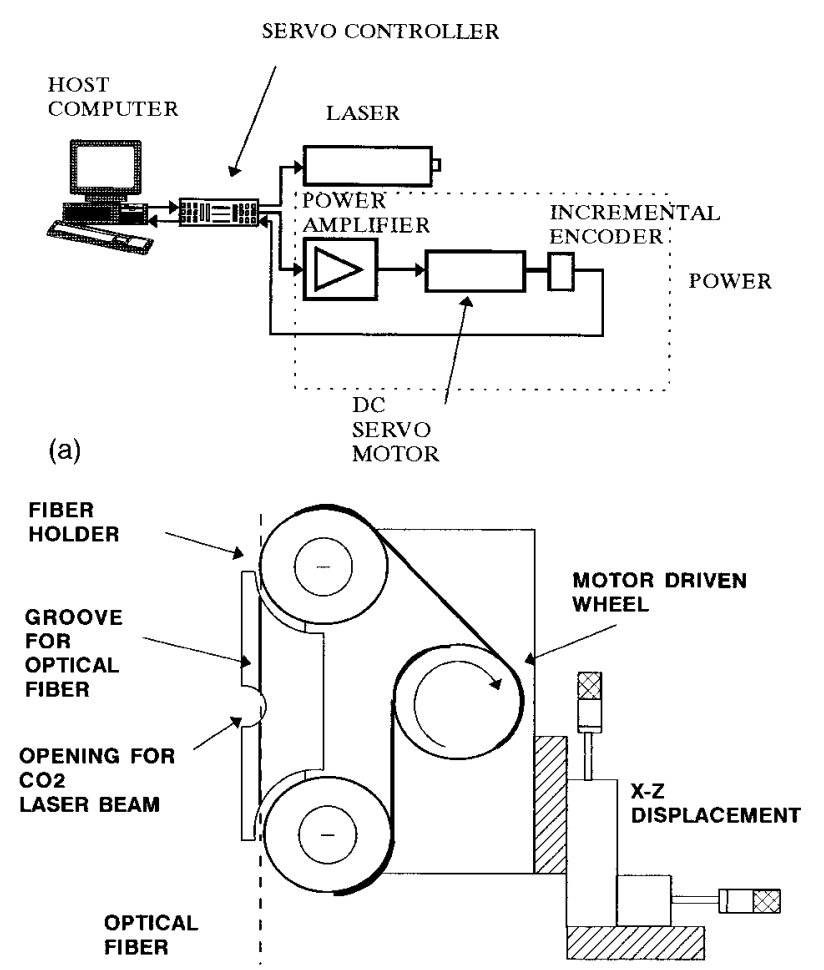

(b)

FIG. 1. (a) Schematic of pulling machine; (b) mechanical layout.

$R^{\prime}-R \cos \theta$, where $\theta$ is the phase angle between the detected oscillation and the voltage excitation, $R^{\prime}$ is the initial amplitude of the tip, and $R$ is the instantaneous amplitude. The reason for this can be seen by reference to Fig. 5. This signal after being linearized by the log amplifier has an approximately linear dependence with the tip-sample distance. If $R \cos \theta$ were used directly, the linearized signal would now have an exponential distance dependence, and such a feedback loop would be highly unstable, and would tend to oscillate. For our experiments, the oscillation amplitude is maintained at between 5 and $10 \mathrm{~nm}$.

\section{RESULTS AND DISCUSSION}

Our SNOM is a reflection-mode microscope. In this article we present results of its characterization, which were obtained on a test cross grating. This is a carbon film containing lines in two perpendicular directions, of period 460 $\mathrm{nm}$, with V-shaped grooves $80 \mathrm{~nm}$ wide and $40 \mathrm{~nm}$ deep (Fig. 6). ${ }^{9}$ Figure 7 shows a typical shear-force image of this grating. The lateral resolution can be inferred to be better than $30 \mathrm{~nm}$. For this image, the oscillation amplitude of the tip was calculated to be 5-10 $\mathrm{nm}$ peak-peak. Resolution

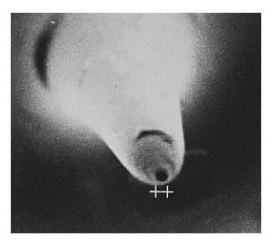

FIG. 2. SEM image of coated SNOM tip, showing aperture at end. Aperture size is $130 \mathrm{~nm}$.

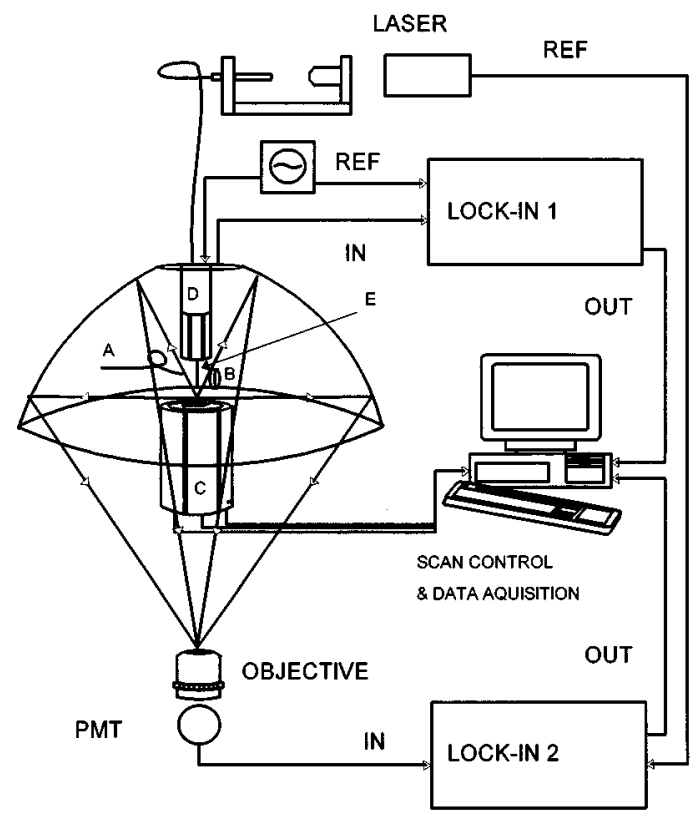

FIG. 3. Schematic of SNOM setup. (a) optical fiber for shear force; (b) photodiode; (c) sample scanner piezo; (d) dither piezo; (e) SNOM fiber tip.

better than $30 \mathrm{~nm}$ was obtained for almost every single tip used. Even metal-coated tips which are at least $250 \mathrm{~nm}$ in size at the apex still reproducibly show such resolution.

For imaging of such a sample containing depressed features, there are at least three distinct regimes:

(1) The tip is small enough to fit inside the depression, and hence maintain a constant distance from the sample-see Fig. 9(a).

(2) the tip is too large to fit in-Fig. 9(b).

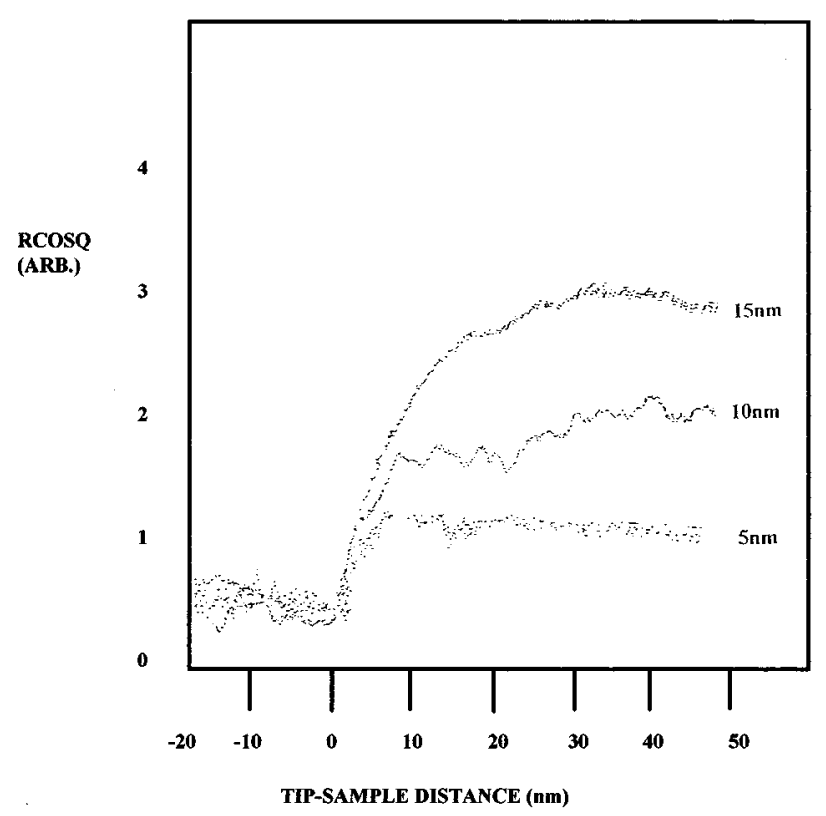

FIG. 4. Shear-force approach curves showing dependence of in-phase fiber oscillation amplitude. $R \cos \theta$, as a function of tip-sample separation for three different oscillation amplitudes. 


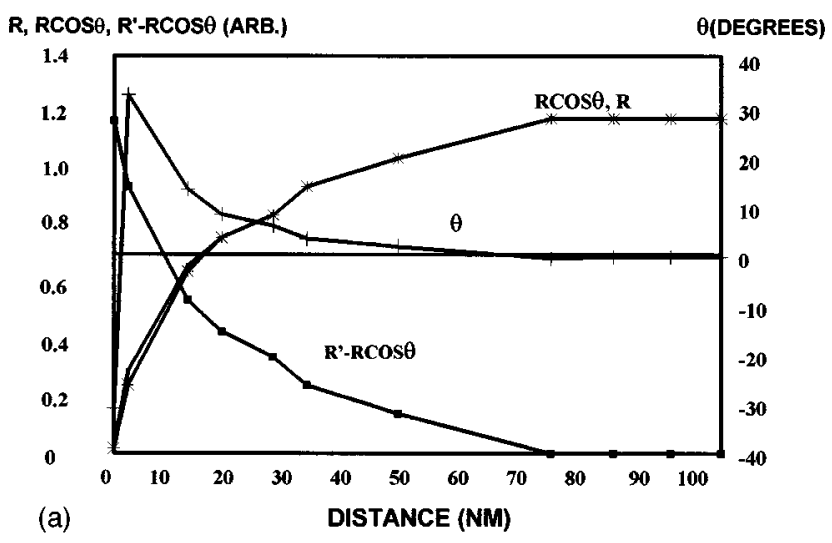

LN(RCos $\theta)$, LN(R'-Rcose) (ARB.)

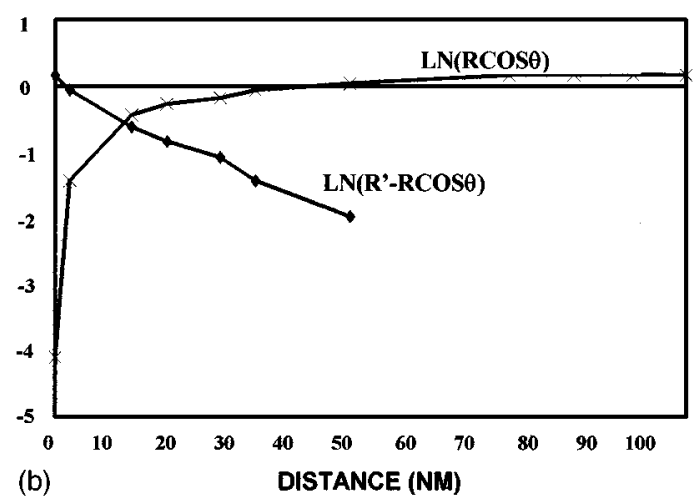

FIG. 5. (a) Distance dependence of shear-force fiber oscillation amplitude $R$; phase $\theta$; in-phase amplitude $R \cos \theta$; and normalized in-phase oscillation amplitude $R^{\prime}-R \cos \theta$; (b) Distance dependence of logarithms of inphase fiber oscillation amplitude, and normalized amplitude.

(3) the tip may be able to partially fit inside, and possibly stick in the depression-Fig. 9(c).

In this work we argue that in this case the feedback may work in the wrong sense. For imaging of the above sample, case (1) may only be realized by a protrusion in the $\mathrm{Al}$ coating on the tip. Since the size of the tip is greater than 250

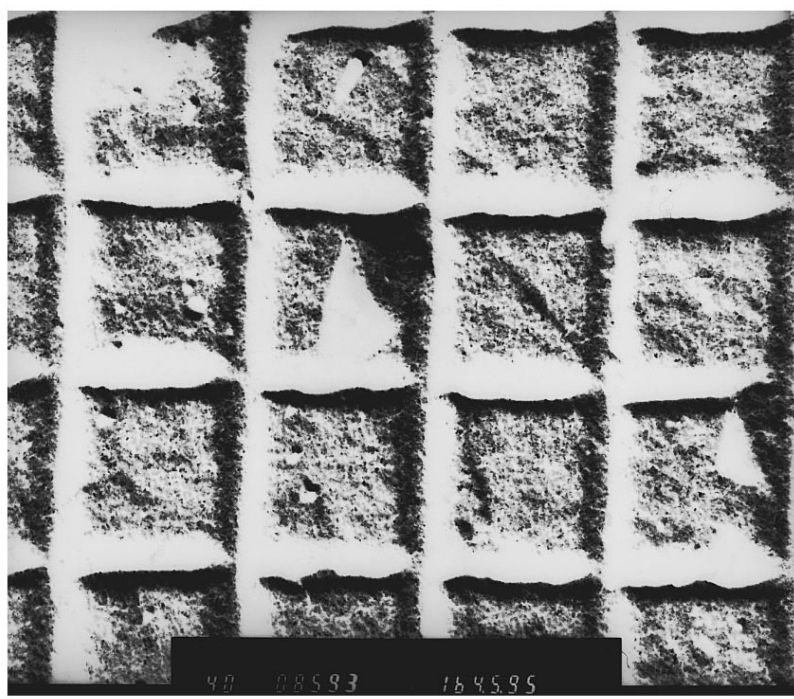

FIG. 6. TEM image of grating sample. Area $=1.8 \mu \mathrm{m}^{2}$.

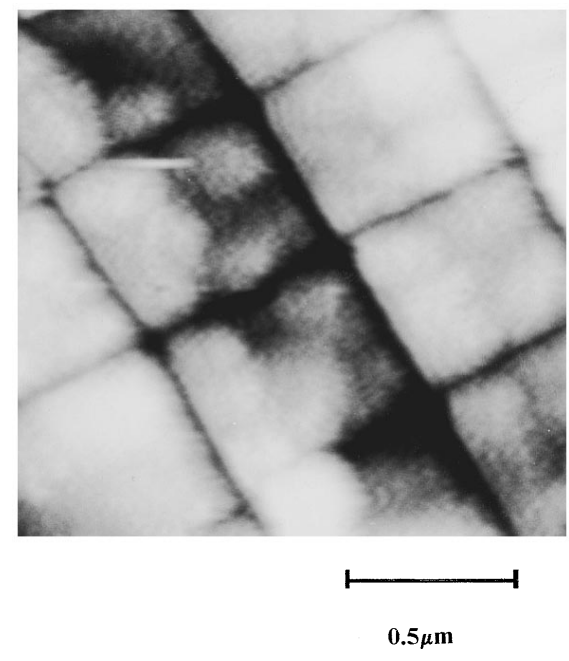

FIG. 7. Shear-force image of grating. Scan size $=1.25 \mu \mathrm{m}^{2}$.

$\mathrm{nm}$, this could mean that while the distance between this protrusion and the sample is constant, the distance between the optical aperture and the sample is not necessarily so. Figure 7 is consistent with this regime. This can be deduced using the fact that the error signal associated with this image showed no features, and the depth of the grooves is consistent with the expected value. Figure 8 is an image of the grating consistent with regime (3). The dither direction of the tip is indicated. There are three interesting features to note:

(i) The grating lines appear to be raised, rather than depressed;

(ii) the lines are somewhat smeared out in the dither direction as is to be expected; and

(iii) the width of the grooves is much larger than $80 \mathrm{~nm}$.

To understand why there appears to be contrast reversal, consider Fig. 9. This represents the scanning of a tip across a groove for the three cases. The essential feature is that when the tip is centered over the groove, it can get stuck, and the feedback loop will tend to withdraw it, whereas when it is slightly off center, it can partially extend into the groove. This is consistent with line profiles of the grooves, as shown

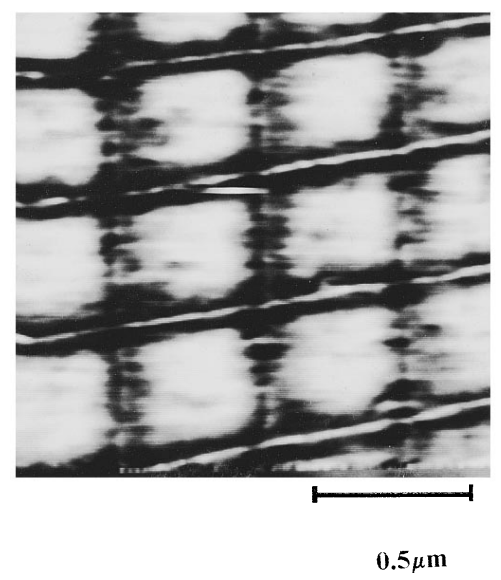

FIG. 8. Shear-force image of grating showing reversed contrast. Scan size $=1.25 \mu \mathrm{m}^{2}$. 


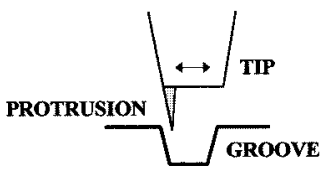

$\mathbf{A}$

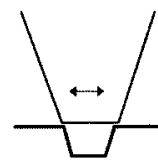

B

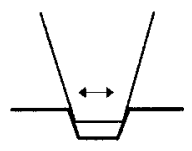

$\mathbf{C}$
FIG. 9. Illustration of different tip-sample size regimes: (a) tip small enough to fit into depressions; (b) tip too large to fit into depressions; and (c) tip only small enough to partially fit into depressions.

in Fig. 10. This becomes more important as the depth of the groove is increased. The lines appear smeared out in the dither direction due to the fact that when the tip is oscillating by $10-20 \mathrm{~nm}$ laterally, the effective lateral distance over which the groove influences the tip becomes $20-40 \mathrm{~nm}$. This is not the case for the perpendicular direction. The grooves may appear to be larger than expected if the tip is large enough-i.e., the edge of the tip may feel the influence due to the groove long before the very apex does.

Another illustration of this is shown in Fig. 11, which is a shear-force image of the grating, again where the lines appear raised. Figure 12 shows a zoom-in into the area indicated in Fig. 11 where there are three depressions about 40 $\mathrm{nm}$ in size, separated by about $60 \mathrm{~nm}$. The hole on the righthand side $(60 \mathrm{~nm})$ is large enough to partially fit the end of the tip, but then it gets stuck when it is centered on the hole, hence the white region at the center, whereas the other two holes $(40 \mathrm{~nm})$ are too small. The reason why the left-handside part of the image of the depression on the right-hand side appears dark in the shear-force and error signal images is due to the dynamic response time of our feedback loop. The scan direction is horizontal, so the tip overshoots on entering the depression, and then again on leaving it. The result is that the measured depth of the depressions is greater than the actual depth. The second image is the error signal.

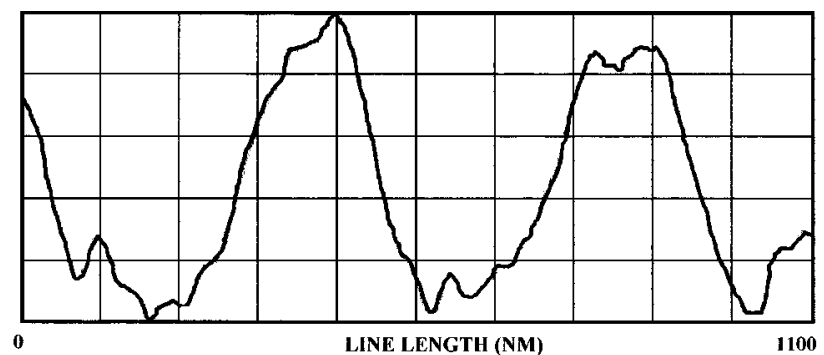

(a)

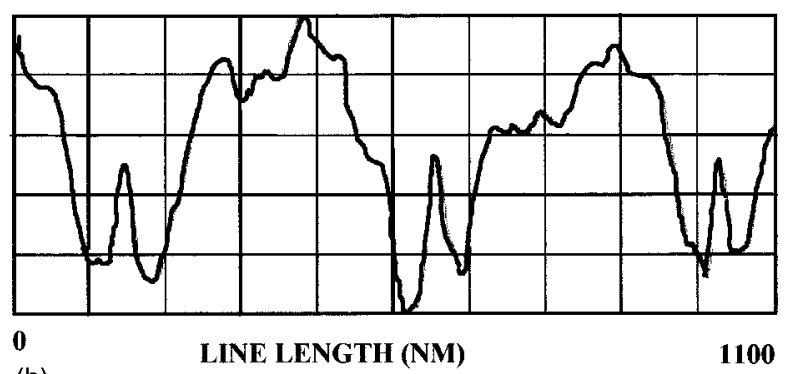

(b)

FIG. 10. Line profiles of grating image in Fig. 8; (a) parallel, and (b) perpendicular to dither direction, showing smoothing out of features perpendicular to dither direction.

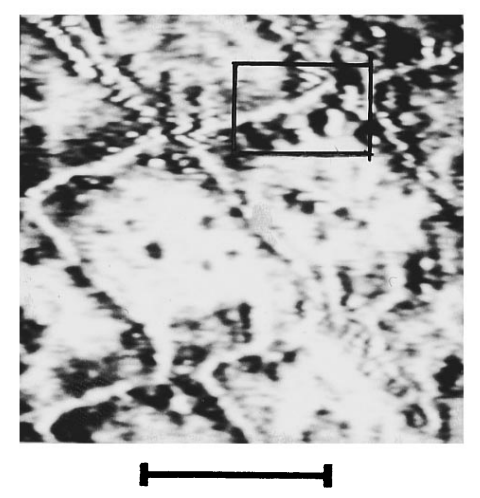

$400 \mathrm{~nm}$

FIG. 11. Shear-force image of grating. Scan size $=930 \times 870 \mathrm{~nm}^{2}$

The third image is the SNOM image obtained of the same area, in the external-reflection geometry. The contrast mechanism is purely topographic, and is very much enhanced by the sharp withdrawal of the tip when centered on the hole, as shadowing of the reflected light by the tip is an extremely important effect. Optical resolution better than 40 $\mathrm{nm}$ is clearly demonstrated in this image: The three holes are just resolved. It was taken with $\lambda=685 \mathrm{~nm}$.

\section{CONCLUSIONS}

It has been demonstrated that, for certain configurations of tip and sample in shear-force microscopy, contrast reversal may occur, e.g., over a depression on the surface, the feedback may withdraw the tip after it gets stuck in the depression. Therefore, the distance between the tip and sample is

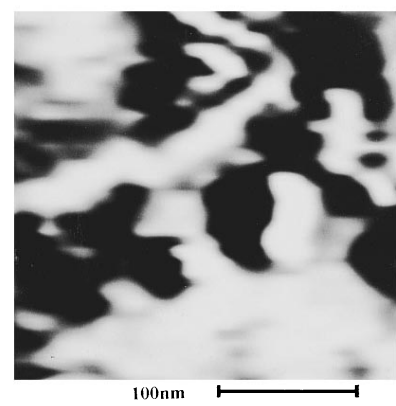

(a)

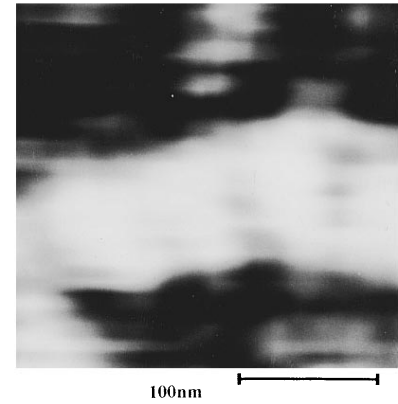

(c)

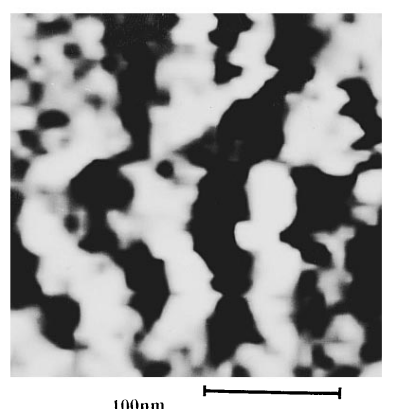

(b)
$100 \mathrm{~nm}$

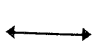

FIG. 12. Zoom-in of area indicated in Fig. 11: (a) shear-force image; (b) error signal image; and (c) SNOM image. 
not always kept constant with shear-force-based feedback. This serves to complicate image interpretation in both shearforce and SNOM images, as there will always be a convolution between topography and optical features. This is particularly important, as the optical aperture and the shear-force tip are rarely, if ever, at the same place.

\section{ACKNOWLEDGMENTS}

Thanks are due to S. Madsen for work done on the original fiber-pulling machine, and to $M$. Viret for helping with tips. Thanks are also due to B. Hecht, D. Pohl, and
L. Novotny for many useful and illuminating discussions while this work was still in its infancy.

${ }^{1}$ E. Betzig, P. L. Finn, and J. S. Weiner, J. Appl. Phys. 60, 2484 (1992).

${ }^{2}$ R. Toledo-Crow, P. C. Yang, Y. Chen, and M. Vaez-Iravani, Appl. Phys. Lett. 60, 2957 (1992).

${ }^{3}$ E. Betzig, J. K. Trautman, T. D. Harris, J. S. Weiner, and R. L. Kostelak, Science 251, 1468 (1991)

${ }^{4}$ G. Binnig, C. F. Quate, and Ch. Gerber, Phys. Rev. Lett. 56, 930 (1986).

${ }^{5}$ Y. Martin, C. C. Williams, and H. K. Wickramasinghe, J. Appl. Phys. 61, 4723 (1987).

${ }^{6}$ E. Betzig, A. Harootunian, A. Lewis, and M. Isaacson, Appl. Opt. 25, 1890 (1986).

${ }^{7}$ I. V. Shvets and S. Madsen, Irish Patent No. S63296, 1995.

${ }^{8}$ For instance, Sutter puller, model P-87.

${ }^{9}$ Agar Scientific transmission electron microscopy test sample no. S-107. 